\title{
Determinants of postoperative abscess occurrence and percutaneous drainage in children with perforated appendicitis
}

\author{
Sherif Emil • Sherif Elkady • Layla Shbat • \\ Fouad Youssef • Robert Baird · Jean-Martin Laberge • \\ Pramod Puligandla $\cdot$ Kenneth Shaw
}

Accepted: 7 October 2014 / Published online: 2 November 2014

(c) Springer-Verlag Berlin Heidelberg 2014

\begin{abstract}
Purpose Postoperative abscesses after perforated appendicitis have no clear risk factors or indications for percutaneous drainage. Our study addressed these two issues.

Methods A logistic regression model was used to delineate risk factors for postoperative abscess in children with perforated appendicitis treated during a recent 5-year period. Drainage of abscess was compared to antibiotic treatment.

Results Postoperative abscess occurred in $42(14.8 \%)$ of 284 patients. Higher WBC count, presence of bowel obstruction at presentation, diffuse peritonitis with a dominant abscess at surgery, and one specific surgeon were significantly associated with postoperative abscess, while fever or pain requiring narcotics at the time of abscess diagnosis was significantly associated with drainage. Compared to non-drainage, those drained had longer hospital stay including readmissions $(15.9 \pm 5.3$ vs. $12.2 \pm 4.6$ days, $p<0.005$ ) and less readmissions ( 9.5 vs. $33.3 \%, p=0.06$ ). Over the 5-year period, there was no increased trend in abscess occurrence $(p=0.56)$, but there was an increased trend in the use of percutaneous drainage $(p=0.02)$.

Conclusions The risk of a postoperative abscess can be predicted by specific clinical characteristics, surgical findings, and treatment-related factors. Percutaneous drainage was associated with longer hospital stays, but less readmissions.
\end{abstract}

Presented at the 17th International Symposium of Pediatric Surgical Research, Toronto, Ontario, Canada, September 22-23, 2014.

S. Emil $(\bowtie) \cdot$ S. Elkady · L. Shbat · F. Youssef · R. Baird ·

J.-M. Laberge $\cdot$ P. Puligandla $\cdot$ K. Shaw

Division of Pediatric General and Thoracic Surgery, The

Montreal Children's Hospital, 2300 Tupper, C-818, Montreal,

QC H3H 1P3, Canada

e-mail: Sherif.Emil@McGill.ca
Keywords Appendicitis - Perforated · Children · Abscess · Risk factors · Percutaneous - Drainage

\section{Introduction}

Appendicitis is the most common acute surgical illness in children and adults, and one of the most frequent conditions treated by pediatric surgeons. The most important determinant of appendicitis outcomes is the stage of disease at presentation. Perforated appendicitis is associated with a significantly longer hospital stay, morbidity, and complications. Large population studies in the USA and Canada have shown that approximately one-quarter of children will present with perforation [1]. Significant variability in care for these children exists between institutions, and even between surgeons within a single institution [2].

Postoperative intra-abdominal abscess is the most common complication of perforated appendicitis and remains a significant problem, ranging in incidence from 3 to $25 \%$ in most large series published since the turn of the century [2-9]. Fike et al. have recently shown that the occurrence of an intra-abdominal abscess doubles the hospital stay and cost of perforated appendicitis [6]. Yet, there is little, if any, consensus regarding the risk factors for intra-abdominal abscess, or the management of this complication. Our study aimed to address these two issues.

\section{Methods}

Patient population

The inpatient and outpatient medical records of all children treated for perforated appendicitis during a recent 5-year 
period, November 1, 2008-August 31, 2013, were retrospectively reviewed. All patients were treated according to a previously published clinical pathway $[2,4]$. In summary, patients received preoperative antibiotics and were continued on postoperative antibiotics until resolution of ileus, fever (temperature $\leq 37.5^{\circ} \mathrm{C}$ ), and leukocytosis. Intraoperative cultures were not sent, and patients were treated empirically with ampicillin, gentamicin or tobramycin, and metronidazole. When symptoms persisted 5-7 days after appendectomy, the patient underwent imaging with ultrasound. The diagnosis of postoperative abscess was made in the context of the ultrasound results and the patient's symptoms. The attending surgeon decided if a drainage procedure was indicated in consultation with the attending interventional radiologist. Follow-up occurred 2 weeks after discharge and further if needed.

Data collection

Data were collected on all patients in four categories.

1. Patient demographics and operating surgeon.

2. Presentation and workup: this included presenting symptoms and duration, physical findings, laboratory results, and imaging results.

3. Operative approach (laparoscopic or open) and findings classified as:
a. localized peritonitis (early perforation)—no abscess;
b. diffuse peritonitis-no dominant abscess;
c. diffuse peritonitis $+\geq 1$ dominant abscess;
d. no diffuse peritonitis-contained abscess;
e. any of the above with free fecalith.

4. Outcomes: hospital stay, wound infection, intra-abdominal infection, and other significant complications.

Additional data were collected on patients who developed intra-abdominal abscesses. These included symptoms at the time of diagnosis of abscess, radiologic findings (single abscess, multiple abscesses, or one or more fluid collections without enhancing rim), basis of decision to drain (if done), duration of drainage, drain-associated complications, duration between drainage or continuation of antibiotics only and discharge, total number of imaging modalities used, and any other pertinent details.

\section{Statistical analysis}

The study population was divided into two subgroups: patient with and without postoperative abscess. Twelve potential determinants of postoperative abscess occurrence [age, year of treatment (2009-2013), gender, attending surgeon, symptom duration, transfer from another institution, bowel obstruction or severe ileus at presentation, white blood cell count, \% neutrophils, operative duration, surgical approach, and operative findings (a-e as described above)] were tabulated in frequencies (for categorical factors) for all subjects and for each of the two subgroups, or were presented using summary statistics (n, median, mean, standard deviation, range) for all subjects and for each of the two subgroups. Chi-square test (for categorical variables) or Wilcoxon rank sum test (for continuous variables) was performed to assess homogeneity of relevant factors between the two subgroups. Multivariable logistic regression analysis was performed to examine the association of the candidate factors, selected from univariate analysis with postoperative abscess.

Patients who developed an abscess or symptomatic fluid collection were divided into two subpopulations: those treated with prolonged antibiotics alone or those treated with antibiotics and a drainage procedure. We first assessed homogeneity of relevant factors/outcomes between the two subpopulations following the same approach used for analysis of postoperative abscess. Multivariable logistic regression analysis was employed to examine the association of relevant factors with treatment (prolonged antibiotics alone or drainage).

The Cochran-Armitage trend test was used to examine for trends in abscess incidence and interventional radiology drainage over the 5-year period.

Continuous variables are reported as mean \pm standard deviation, unless specifically mentioned. All analyses were performed using SAS 9.3 (SAS Institute, Cary NC). A $p$ value $<0.05$ was considered to be statistically significant.

\section{Study approval}

The study was approved by the Pediatric Research Ethics Board of the McGill University Health Centre Research Institute (13-294-PED).

\section{Results}

Patient cohort

During the study period, 1,145 appendectomies were performed for a preoperative diagnosis of acute appendicitis. These included 297 patients with perforated appendicitis, a perforation rate of $26 \%$. Thirteen patients were treated with a primary nonoperative approach and were excluded, leaving a study cohort of 284 patients. Patient with gangrenous, non-perforated, appendicitis were not included in the study since they have a different clinical trajectory [10]. 
The mean age was $10.0 \pm 3.9$ years. Males comprised $63 \%$. The patients were operated by six surgeons. With the exception of one part-time surgeon who performed nine appendectomies, the volume of the other five surgeons was in a close range, 44-65. A preoperative ultrasound was performed in $62.6 \%$ of patients. It was positive in $64.5 \%$, negative in $12.9 \%$, and indeterminate in $22.5 \%$. Preoperative CT scans were done in only $7 \%$ of patients and were all positive. The surgical approach was laparoscopic in 231 cases $(81 \%)$, open in 44 cases $(15 \%)$, and laparoscopic converted to open in 10 cases $(4 \%)$. Eight of the ten conversions were by a single surgeon. Four wound infections were documented $(1.4 \%)$. Eleven patients $(3.9 \%)$ experienced non-infectious major complications, including adhesive small bowel obstruction in 5, prolonged ileus in 3, cardiac arrhythmia in 2, and gangrenous bowel and wound dehiscence in 1 . Initial hospital stay was $7.0 \pm 4.3$ days. Twenty-one patients $(7.4 \%)$ were readmitted for $3.5 \pm 2.5$ days.

Follow-up after discharge was documented in 271 cases $(95.4 \%)$ at a median of 14 days and range of 2-361 days.

\section{Determinants of postoperative abscess}

A postoperative abscess was diagnosed in 42 patients $(14.8 \%)$ at a median of 8 days after appendectomy (range 5-14 days). Ultrasound was the diagnostic modality in all but three patients, who underwent CT scan. Compared to patients without an abscess, those with an abscess had significantly higher incidences of wound infection (4.8 vs. $0.8 \%, p=0.046$ ), non-infectious major complications (11.9 vs. $2.5 \%, p=0.003$ ), and readmissions (26.2 vs. $3.7 \%, p<0.001)$. Patients with an abscess also had significantly longer initial hospital stays $(13.2 \pm 5.7$ vs. $5.9 \pm 2.8$ days, $p<0.001)$ and total hospital stays including readmissions $(14.1 \pm 5.3$ vs. $6.0 \pm 3.0$ days, $p<0.001$ ).

Univariate analysis of the 12 potential predictors of abscess occurrence is shown in Table 1. Patients who developed postoperative abscess had longer symptom duration, higher prevalence of bowel obstruction or severe ileus at presentation, longer operative duration, a higher prevalence of diffuse peritonitis with dominant abscess at initial surgery, and a higher incidence of free fecalith at initial surgery. Incidence of postoperative abscess by attending surgeon varied from 9.1 to $24.1 \%$, but was not statistically significant on univariate analysis $(p=0.17)$.

Multivariate analyses showed that higher WBC count $[\mathrm{OR}=1.08,95 \%$ CI 1.01-1.16), $p=0.02]$, presence of bowel obstruction or severe ileus at presentation $[\mathrm{OR}=6.0$, $95 \%$ CI 2.2-20.0, $p=0.001]$, and one specific surgeon $[\mathrm{OR}=5.5,95 \%$ CI 1.2-23.9), $p=0.02]$ were significantly associated with postoperative abscess occurrence.
Table 1 Univariate analysis of potential predictors of abscess occurrence

\begin{tabular}{|c|c|c|c|}
\hline Variable & Abscess & No abscess & $p$ \\
\hline $\mathrm{N}$ & 42 & 242 & \\
\hline Age (years) & $11.0 \pm 4.3$ & $10.4 \pm 3.8$ & 0.31 \\
\hline Gender ( $\%$ male $)$ & 59.5 & 64.0 & 0.57 \\
\hline Symptom duration (days) & $4.0 \pm 2.2$ & $3.0 \pm 2.7$ & $<0.001$ \\
\hline $\begin{array}{l}\text { Transfer from another } \\
\text { facility }(\%)\end{array}$ & 19.0 & 20.7 & 0.81 \\
\hline $\begin{array}{l}\text { Bowel obstruction or severe } \\
\text { ileus@presentation }(\%)\end{array}$ & 23.8 & 7.4 & 0.001 \\
\hline WBC count $(1,000 / \mathrm{ul})$ & $19.9 \pm 6.8$ & $17.3 \pm 5.3$ & 0.06 \\
\hline Operative duration (minutes) & $76.9 \pm 36.7$ & $63.9 \pm 23.8$ & 0.04 \\
\hline $\begin{array}{l}\text { Surgical approach (\% } \\
\text { laparoscopic) }\end{array}$ & 88.1 & 79.8 & 0.44 \\
\hline $\begin{array}{l}\text { Diffuse peritonitis with } \\
\text { dominant abscess } \\
\text { at surgery }(\%)\end{array}$ & 19.0 & 7.9 & 0.02 \\
\hline Free fecalith at surgery $(\%)$ & 35.7 & 14.0 & 0.001 \\
\hline
\end{tabular}

Comparison of drainage to antibiotics alone

Twenty-one of 42 patients with abscess $(50 \%)$ underwent drainage. Nineteen of those $(90.5 \%)$ underwent imageguided percutaneous drainage by interventional radiology and 2 underwent operative drainage. The comparison of patient characteristics, imaging findings, and outcomes between the drainage and non-drainage groups is shown in Table 2. Treatment failure was identified when a subsequent procedure was required. Of patients initially treated with antibiotics alone, $18(85.7 \%)$ were discharged without further interventions, $2(9.5 \%)$ proceeded to percutaneous drainage, and $1(4.8 \%)$ proceeded to operative drainage. Of patients who underwent initial percutaneous drainage, one $(4.8 \%)$ required subsequent operative drainage. All four patients who underwent operative drainage required no further interventions.

Multivariate analysis showed that only persistent fever $[\mathrm{OR}=25.4,95 \%$ CI 1.4-454.0, $p$ value $=0.03]$ and pain requiring narcotics at the time of abscess diagnosis $[\mathrm{OR}=12.8,95 \%$ CI $1.2-37.4, p$ value $=0.03]$ were significantly associated with the use of drainage. All the differences in imaging findings that were significant on univariate analysis were no longer significant on multivariate analysis.

Study of trends

Over the 5-year period, the rates of postoperative abscess varied from a low of $10.3 \%$ in 2011 to a high of $21.9 \%$ in 2013, with no significant trend in abscess incidence $(p=0.56)$. The rate of abscess drainage varied from a low 
Table 2 Comparison of patients who underwent drainage to those who did not

\begin{tabular}{|c|c|c|c|}
\hline Variable & Drainage & No drainage & $p$ \\
\hline$N$ & 21 & 21 & \\
\hline \multicolumn{4}{|l|}{ Patient characteristics } \\
\hline Age (years) & $12.8 \pm 3.5$ & $9.3 \pm 4.4$ & 0.01 \\
\hline $\begin{array}{l}\text { Fever at the time of } \\
\text { abscess diagnosis }(\%)\end{array}$ & 100 & 66.7 & 0.004 \\
\hline $\begin{array}{l}\text { Ileus at the time of } \\
\text { abscess diagnosis }(\%)\end{array}$ & 47.6 & 33.3 & 0.35 \\
\hline $\begin{array}{l}\text { Diarrhea at the time of } \\
\text { abscess diagnosis }(\%)\end{array}$ & 9.5 & 19.0 & 0.38 \\
\hline $\begin{array}{l}\text { Pain requiring narcotics at } \\
\text { the time of abscess } \\
\text { diagnosis }(\%)\end{array}$ & 81.0 & 28.6 & 0.001 \\
\hline \multicolumn{4}{|l|}{ Imaging findings } \\
\hline $\begin{array}{l}\text { Incidence of single } \\
\text { abscess }(\%)\end{array}$ & 14.3 & 42.9 & 0.03 \\
\hline $\begin{array}{l}\text { Diameter of largest } \\
\text { abscess }(\mathrm{cm})\end{array}$ & $8.3 \pm 1.8$ & $5.5 \pm 2.3$ & 0.001 \\
\hline $\begin{array}{l}\text { Total volume of all } \\
\text { abscesses }(\mathrm{ml})\end{array}$ & $316.4 \pm 232.6$ & $54.3 \pm 65.0$ & $<0.001$ \\
\hline $\begin{array}{l}\text { Distended bowel on } \\
\text { imaging }(\%)\end{array}$ & 33.3 & 33.3 & 1.00 \\
\hline Fecalith on imaging (\%) & 33.3 & 9.5 & 0.06 \\
\hline \multicolumn{4}{|l|}{ Outcomes } \\
\hline $\begin{array}{l}\text { Duration between abscess } \\
\text { diagnosis and discharge } \\
\text { (days) }\end{array}$ & $8.4 \pm 4.2$ & $5.6 \pm 4.3$ & 0.005 \\
\hline Initial hospital stay (days) & $15.6 \pm 5.1$ & $11.1 \pm 5.2$ & 0.003 \\
\hline $\begin{array}{l}\text { Total hospital stay } \\
\text { including readmissions } \\
\text { (days) }\end{array}$ & $15.9+5.4$ & $12.2+4.6$ & 0.01 \\
\hline Readmissions (\%) & 9.5 & 33.3 & 0.06 \\
\hline
\end{tabular}

of $18.1 \%$ in 2009 to a high of $71.4 \%$ in 2013, demonstrating increased trend in the use of percutaneous drainage $(p=0.02)$.

\section{Discussion}

Our surgical group has been interested in the outcomes of pediatric appendicitis for the past quarter century and has helped create clinical pathways and standards for treatment of this common disease [2, 4, 10-15]. Our rates of postoperative abscesses after perforated appendicitis have been consistently below $10 \%$, quite favorable when compared to published standards [3-9, 11, 14]. Recently, we noticed an increase in this complication, as well as a higher incidence of drainage of such abscesses by interventional radiology. This led to the present analysis.
We found that the abscess rate in our practice has nearly doubled compared to a decade previously, offering the first lesson from this study [11]. Clinical pathways have to be regularly monitored and audited to confirm continued validity and effectiveness. Despite this increase in abscess rate, average initial hospital stay for all perforated patients remained stable at approximately 7 days. This was probably due to increased discharge of selected patients who have persistent leukocytosis, but no ileus or fever, on oral antibiotics.

Associations with abscess recurrence on univariate analysis included mostly patient factors such as symptom duration, a clinical picture of bowel obstruction on presentation, diffuse peritonitis with abscess found at surgery, and a free fecalith found at surgery. Bowel obstruction persisted in the multivariate analysis. Some of these findings were also seen in the few previous studies that looked at the same issue of abscess determinants. We have previously highlighted the role of a retained fecalith in abscess formation and persistence, a concept also described in the radiology literature $[4,16]$. In a multi-institutional study, Henry et al. found that a fecalith and diarrhea at presentation (often a harbinger of a pelvic abscess) were associated with postoperative abscess [5]. Using data from several clinical trials, Fike et al. also found that diarrhea at presentation and the presence of a fecalith were associated with a higher chance of postoperative abscess [6]. Retrieval of a fecalith seen either on preoperative imaging or discovered during operation is therefore of paramount importance in abscess prevention. A higher body mass index was also a risk factor [6].

In our study, a longer surgical time was associated with abscess formation. This is likely a surrogate for a more difficult operation. Most interestingly, we found that one of the six surgeons had a significantly higher rate of abscess formation on the multivariate analysis that adjusted for other risk factors. This surgeon actually had the third highest volume of perforated appendicitis in the group of six. Therefore, there may be a role for technical modifications in reducing abscess formation.

Multiple other therapeutic attempts have been made to decrease postoperative abscess formation in perforated appendicitis patients. Unfortunately, almost none of these have proven effective. Placement of drains at operation, a component of early protocols, has actually been shown to increase abscess formation and other complications in multiple studies and has been essentially abandoned [17, 18]. Prolonged antibiotic treatment after resolution of symptoms, insistence on a minimal duration of antibiotics, or using one antibiotic regimen versus another are all ineffective $[3,6,19]$. We have always stopped antibiotics according to clinical criteria, without any minimum treatment periods. The reported higher rates of abscess 
formation after laparoscopic appendectomy have been contradicted by recent series and probably reflected a learning curve in the early experience $[8,14]$. Populationbased studies have shown a steady increase in the use of laparoscopic approach for both simple and perforated appendicitis [20].

Irrigation of the abdominal cavity, a long-held surgical dictum, has not proven effective in a recent clinical trial of irrigation versus suction alone, in which both study arms had abscess rates of approximately $19 \%$ [7]. Tailoring antibiotic regimens to intraoperative cultures has not been associated with improved outcomes or decreased abscess formation, leading most investigators to abandon the practice [21-24]. With few exceptions (e.g., immunocompromised patient, child already taking antibiotics), our service stopped culturing pus from perforated appendicitis in the mid 1990s. Fallon has recently challenged this practice, showing significant emergence of organisms such as Pseudomonas, Enterococcus, and Escherichia coli resistant to the typical antibiotic regimens [9]. However, the abscess rate was similar in the cohorts studied before and after routine cultures were sent, and no resistant organisms were cultured from drained abscesses [9]. Nevertheless, the authors felt that tailoring antibiotic therapy may be of benefit in reducing postoperative abscesses in their future experience [9]. One should remember that the data arguing against routine cultures comes mostly from studies 10-20 years old [21-24]. However, recently published guidelines by the Surgical Infection Society for diagnosis and treatment of complicated intra-abdominal infection in children support response-guided antibiotic treatment, rather than tailoring treatment to cultures, as well as limiting the duration to 7 days in patients whose peritonitis has resolved [25].

Ein et al. investigated a potential role for routine imaging on the fifth postoperative day in predicting abscess after appendectomy, but found that radiologic findings were only useful if they correlated with clinical symptoms [26]. This is an important concept, since fluid collections after appendectomy are not unusual. Early imaging may lead to unnecessary interventions. Decisions to treat the patient for an abscess complication have to interpret imaging in the clinical context.

One of the few interventions that have been shown to be of benefit is the enactment of clinical pathways for treatment of perforated appendicitis, a practice recommended by the Surgical Infection Society [2-4, 25]. These pathways prevent variability in care within a single institution and between institutions and allow for outcome evaluation and improvement.

The second aim of our study was to compare the cohort of patients treated with antibiotics alone to those who underwent drainage. This was strictly an observational goal, as the decision to drain was left completely to the attending surgeon and was not guided by any specific protocol or radiologic findings. Drainage increased markedly in our institution over the past 5 years, as pediatric interventional radiology became increasingly available. Not surprisingly, patients who underwent drainage were more symptomatic, with a higher percentage having persistent pain and fever. The differences in abscess size and total volume between drained and non-drained patients did not persist on multivariate analysis, possibly due to the small sample size. Drainage was associated with prolonged initial hospital stay, but less readmissions. However, most patients readmitted after antibiotic treatment alone had very short hospital stays of 1-2 days. All patients recovered completely after either treatment modality.

One of the earliest publications on drainage of postoperative abscess by interventional radiology techniques came from the Hospital for Sick Children in Toronto, a leader in pediatric interventional radiology [27]. This early series established the feasibility and safety of these procedures, reporting a success rate of $91 \%$ and complication rate of $2 \%$ [27]. However, in the last 20 years, a plethora of reports encouraged the practice of treating postoperative abscesses with antibiotics only without drainage procedures, reporting success rates of 80-90\% [28-33]. Unfortunately, all these reports constituted extremely low level evidence, as they were all observational studies with few patients, no attempts to control for abscess size or symptoms, and frequently poor reporting of overall outcomes. In addition, the duration to abscess resolution was frequently long, reported to be a median of 18.5 days with a range of 7-60 days in one study [30]. Recent analyses similar to ours have yielded similar results, showing longer hospital stays, but less readmission after drainage procedures [34, 35]. Using volume measurements, it has been suggested that draining an abscess measuring less than 20-100 $\mathrm{ml}$ may not incur any additional benefits [34, 35]. However, these data again come from uncontrolled retrospective studies. The question of whether antibiotics alone versus drainage procedures are most effective for postoperative abscesses begs for a multi-center randomized trial that starts with a clear definition of what constitutes an abscess, a definition lacking from the most recent Surgical Infection Society recommendations [25].

We plan to use the data presented here for two purposes. First, we will make modifications to our clinical pathway to standardize the operative procedure including retrieval of fecaliths, revert to sending intraoperative cultures to study the current flora of perforated appendicitis in our community, and change antibiotic regimens in specific circumstances. We will prospectively audit our modified protocol with the aim of reducing our abscess rate by $50 \%$. Second, we will create standard timelines for imaging patients who 
remain symptomatic, work with our radiologists to create standard definitions and measurements of postoperative abscesses, and use specific criteria to choose drainage versus antibiotic treatment only. We hope to report our future experience with the aim of aiding pediatric surgeons in using these modalities to optimize outcomes.

Acknowledgments We would like to thank Dr. Xianming Tan of the Biostatistics Core Facility, McGill University Health Centre Research Institute for providing assistance with the statistical analyses.

Conflict of interest None.

\section{References}

1. Cheong LHA, Emil S (2014) Outcomes of pediatric appendicitis: an international comparison of the United States and Canada. JAMA Surg 149:50-55

2. Emil S, Taylor M, Ndiforchu F, Nguyen N (2006) What are the true advantages of a pediatric appendicitis clinical pathway? Am Surg 72:885-889

3. Fishman SJ, Pelosi L, Klavon SL, O’Rourke EJ (2000) Perforated appendicitis: prospective outcome analysis for 150 children. J Pediatr Surg 35:923-926

4. Emil S, Laberge J-M, Mikhail P, Baican L, Flageole H, Nguyen L, Shaw K (2003) Appendicitis in children: a ten-year update of therapeutic recommendations. J Pediatr Surg 38:236-242

5. Henry MCW, Walker A, Silverman BL, Gollin G, Islam S, Sylvester K, Moss L (2007) Risk factors for the development of abdominal abscess following operation for perforated appendicitis in children. Arch Surg 142:236-241

6. Fike FB, Mortellaro VE, Juang D, Sharp SW, Ostlie DJ, St. Peter S (2011) The impact of postoperative abscess formation in perforated appendicitis. J Surg Res 170:24-26

7. St Peter SD, Adibe OO, Iqbal CW, Fike FB, Sharp SW, Juang D, Lanning D, Murphy JP, Andrews WS, Sharp RJ, Snyder CL, Holcomb GW, Ostlie DJ (2012) Irrigation versus suction alone during laparoscopic appendectomy for perforated appendicitis. Ann Surg 256:581-585

8. Nataraja RM, Teague WJ, Galea J, Moore L, Haddad MJ, Tsang T, Khurana S, Clarke SA (2012) Comparison of intraabdominal abscess formation after laparoscopic and open appendicectomies in children. J Pediatr Surg 47:317-321

9. Fallon SC, Hassan SF, Larimer EL, Rodriguez JR, Brandt ML, Wesson DE, Palazzi DL, Lopez ME (2013) Modification of an evidence-based protocol for advanced appendicitis in children. J Surg Res 185:273-277

10. Emil S, Gaied F, Lo A, Laberge JM, Puligandla P, Shaw K, Baird R, Bernard C, Blumenkrantz M, Nguyen V-H (2012) Gangrenous appendicitis in children: a prospective evaluation of definition, bacteriology, histopathology, and outcomes. J Surg Res 177:123-126

11. Neilson IR, Laberge JM, Nguyen LT, Moir C, Doody D, Sonnino RE, Youssef S, Guttman FM (1990) Appendicitis in children: current therapeutic recommendations. J Pediatr Surg 25:1113-1116

12. Taylor M, Emil S, Nguen N, Ndiforchu F (2005) Emergent vs urgent appendectomy in children: a study of outcomes. J Pediatr Surg 40:1912-1915

13. Emil SGS, Taylor MB (2007) Appendicitis in children treated by pediatric versus general surgeons. J Am Coll Surg 204:34-39
14. Taqi E, Al Hadher S, Ryckman J, Su W, Aspirot A, Puligandla P, Flageole H, Laberge JM (2008) Outcome of laparoscopic appendectomy for perforated appendicitis in children. J Pediatr Surg 43:893-895

15. Cheong LHA, Emil S (2014) Determinants of appendicitis outcomes in Canadian children. J Pediatr Surg 49:777-781

16. Singh AK, Hahn PF, Gervais D, Vijayraghavan G, Mueller PR (2008) Dropped appendicolith: CT findings and implications for management. AJR 190:707-711

17. Narci A, Karaman I, Karaman A, Erdogan D, Cavusoglu YH, Aslan MK, Cakmak O (2007) Is peritoneal draiange necessary in childhood peforated appendicitis?-A comparative study. J Pediatr Surg 42:1864-1868

18. Allemann P, Probst H, Demartines N, Schafer M (2011) Prevention of infectious complications after laparoscopic appendectomy for complicated acute appendicitis - the role of routine abdominal drainage. Pediatr Surg Int 396:63-68

19. Van Wijck K, de Jong JR, van Heurn WE, van der Zee DC (2010) Prolonged antibiotic treatment does not prevent intra-abdominal abscesses in perforated appendicitis. World J Surg 34:3049-3053

20. Cheong LHA, Emil S. Pediatric laparoscopic appendectomy: a population-based study of trends, associations, and outcomes. J Pediatr Surg. In press.

21. Mosdell DM, Morris DM, Fry DE (1994) Peritoneal cultures and antibiotic therapy in pediatric perforated appendicitis. Am J Surg 167:313-316

22. Bilik R, Burnweit C, Shandling B (1998) Is abdominal cavity culture of any value in appendicitis? Am J Surg 175:267-270

23. Kokoska ER, Silen ML, Tracy TF, Dillon PA, Kennedy DJ, Cradock TV, Weber TR (1999) The impact of intraoperative culture on treatment and outcome in children with perforated appendicitis. J Pediatr Surg 34:749-753

24. Celik A, Ergun O, Ozcan C, Aldemir H, Balik E (2003) Is it justified to obtain routine peritoneal fluid cultures during appendectomy in children? Pediatr Surg Int 19:632-634

25. Solomkin JS, Mazuski JE, Bradley JS et al (2010) Diagnosis and management of complicated intra-abdominal infection in adults and children : guidelines by the surgical infection society and the infectious diseases society of America. Surg Infect 11:79-109

26. Ein SH, Wales P, Langer JC, Daneman A (2008) Is there a role for routine abdominal imaging in predicting postoperative intraabdominal abscess formation after appendectomy for pediatric ruptured appendix? Pediatr Surg Int 24:307-309

27. Jameson DH, Chait PG, Filler R (1997) Interventional drainage of appendiceal abscess in children. AJR 169:1619-1622

28. Gorenstein A, Gewurtz G, Serour F, Somekh E (1994) Postappendectomy intra-abdominal abscess: a therapeutic approach. Arch Dis Child 70:400-402

29. Heloury Y, Baron M, Bourgoin S, Wetzel O, Lejus C, Plattner V (1994) Medical treatment of postappendectomy intraperitoneal abscesses in children. Eur J Pediatr Surg 5:149-151

30. Okoye BO, Rampersad B, Marantos A, Abernethy LJ, Losty PD, Lloyd DA (1998) Abscess after appendicectomy in children: the role of conservative management. Br J Surg 85:1111-1113

31. Dobremez E, Lavrand F, Lefevre Y, Boer M, Bondonny J-M, Vergnes P (2003) Treatment of post-appendectomy intraabdominal deep abscesses. Eur J Pediatr Surg 13:393-397

32. Forgues D, Habbig S, Diallo AF, Kaifa N, Lopez M, Allal H, Guibal MP, Sabatier-Laval E, Galifer RB (2007) Post-appendectomy intra-abdominal abscesses-can they successfully be managed with the sole use of antibiotic therapy? Eur J Pediatr Surg 17:104-109

33. Dhaou MB, Ghorbel S, Chouikh T, Charieg A, Nouira F, Ben Khalifa S, Khemakhem R, Jlidi S, Chaouachi B (2010) Conservative management of post-appendicectomy intra-abdominal abscesses. Ital J Pediatrics 36:68-71 
34. Piper HG, Derinkuyu B, Koral K, Perez EA, Murphy JT (2011) Is it necessary to drain all postoperative fluid collections after appendectomy for perforated appendicitis? J Pediatr Surg 46:1126-1130
35. Gasior AC, Knott EM, Ostlie DJ, St Peter SD (2013) To drain or not to drain: an analysis of abscess drains in the treatment of appendicitis with abscess. Pediatr Surg Int 29:455-458 